\title{
Torsional vibration of carbon nanotubes under initial compression stress
}

\author{
M.M. Selim* \\ Department of Natural and Applied Sciences, AL-Aflaj community College, \\ King Saud University, Al-Aflaj 710-11912 Saudi Arabia
}

(Received on 8 October, 2009)

\begin{abstract}
This study examines torsional vibration of Single-walled carbon nanotubes (SWCNTs) subjected to initial compression stresses. The nanotube structures are treated within the multilayer thin shell approximation with the elastic properties taken to be those of the graphene sheet. Simplified Flügge shell equations of motion are proposed as the governing equations of vibration for the carbon nanotubes. A new equation of motion and phase velocity of torsional waves propagating in carbon nanotubes (CNTs) subjected to initial compression stresses have been derived. The study reveals that the initial stresses present in the tube has a notable effect on the propagation of torsional waves. The results has been discussed and shown graphically. This investigation is very significant for potential application and design of nanoelectronics and nanodevices.
\end{abstract}

Keywords: carbon nanotubes, torsional vibration, initial compression stress, Flügge equations of motion.

\section{INTRODUCTION}

The discovery of carbon nanotubes (CNTs) in 1991 [1] has stimulated ever-broader research activities in science and engineering devoted entirely to carbon nanostructures and their applications. This is due in large part to the combination of their expected structural perfection, small size, low density, high stiffness, high strength(the tensile strength of the outer most shell of MWCNT is approximately 100 times greater than that of aluminum), and excellent electronic properties [2]. As a result, carbon nanotubes (CNT) may find use in a wide range of applications in material reinforcement, field emission panel display, chemical sensing, drug delivery, and nanoelectronics. Despite the potential impact of carbon nanotubes in many areas of science and industry, a robust understanding of their mechanical behavior is lacking and thus limits the design and optimization of CNTenhanced materials. The deformation behavior of CNTs has been the subject of numerous experimental, molecular dynamics (MD), and elastic continuum modeling studies. Experiments at this length scale are themselves still under development and thus have resulted in a range of reported values for various mechanical properties [3]. Furthermore, consistent interpretation of tube geometry when reducing data to properties remains an important issue, particularly when studying multi-wall nanotubes (MWNTs).

Vibrations of CNTs are of considerable importance in a number of nanomechanical devices such as oscillators, charge detectors, clocks, field emission devices and sensors. In addition, CNT vibrations occur during certain manufacturing processes (e.g., ultrasonication in nanocomposite processing) and as part of some nondestructive evaluation processes (e.g., Raman spectroscopy). Electron microscope observations of vibrating CNTs have also been used to indirectly and nondestructively determine the effective elastic moduli and other aspects of mechanical behavior of the CNTs. Microwave excitation has been found to cause intense heating of CNTs. So there is considerable motivation for studying vibration characteristics of CNTs. The CNTs acting as basic elements of nano-structures often occur in

${ }^{*}$ Electronic address: msalimeksu.edu.sa initial stresses due to thermal stress, mismatch between different materials or initially external axial load.

Recently, the vibration of multi wall carbon nanotubes (MWCNTs) and wave propagation of double-wall carbon nanotubes (DWCNTs) have been studied based on Flügge shell equation $[4,5,6]$. These works show that the CNTs have the vibration and sound wave frequency over terahertz because of their nanoscale, which opens a new topic on wave characteristics. Some researches have taken the effect of initial stress on the wave propagation in CNTs [7-11] to understand the dynamic behavior of CNTs.

Although much information is available on vibrational of surface waves in CNTs, the torsional vibrational has not drawn much attention and very little literature is available on propagation of this wave. Lord Rayleigh [12], in his remarkable paper, showed that the isotropic homogenous elastic half-space does not allow a torsional surface wave to propagate. Bhattacharya [13] has been investigated the torsional wave propagation in a two-layered circular cylinder with imperfect bond. The propagation of torsional wave in a finite piezoelectric cylindrical shell has been discussed by Paul et al. [14]. Recently, the propagation of torsional wave in an initially stressed cylinder has been discussed by Dey et al. [15] and Selim [16].

The purpose of this article is to study effects of initial compression stress on torsional vibrational analysis of Singlewalled carbon nanotubes (SWCNTS). The analysis is based on Flügge shell equation. A new equation of motion and phase velocity of torsional waves propagating in CNTs subjected to initial compression stresses have been derived. The study reveals that the initial stresses present in the tubes has strong effect on the propagation of torsional wave in CNTs. Derive program version 6 was used to simplify the Algebraic expressions and the results have been discussed and shown graphically.

The paper is organized as follows. Section 2 provides the governing equation of torsional vibrational in the tubes under initial compression stress. Sections 3 and 4 provide torsional vibration of carbon nanotube with and without initial compression stress. Section 5 presents phase velocity of waves propagate in torsion. Section 6 presents numerical results from special model of CNTs under the effect of compression stress. The results are shown graphically for different values of initial compression stress parameter and thicknesses of 
tubes. Finally, some conclusions on the developed methodology are given in Section 7.

\section{GOVERNING EQUATIONS}

The cylindrical coordinates system used for describing the vibration of carbon nanotubes under initial compression stress and is defined in Fig. 1. Approximate Flügge shell equations are proposed as the governing equations of the problem. In the derivation of Flügge equations, the following assumptions (Love's first approximation) for cylindrical shells are made:

1- All points that lie on a normal to a middle surface before deformation do the same after deformation.

2- Displacements are small compared to the shell thickness.

3- The normal stresses in the thickness direction are negligible (planar state of stress).

In particular, the first assumption may not correspond to reality in the neighborhood of the shell boundaries. However, this fact will not be considered here.

In the sequel, the $x$ coordinate is taken in the axial direction of the shell, where the $\theta$ and $z$ coordinates are in the circumferential and radial directions, respectively (Fig. 1). The displacements of the nanotube are defined by $u_{x}, u_{\theta}$ and $u_{z}$ in the direction of $x, \theta$ and $z$ axes, respectively. $z$ is the displacement from the middle surface of the tube. The coordinates $u_{x}$ and $u_{\theta}$ represent in-plane axial and circumferential displacements of the tube wall midsurface, respectively, and $u_{z}$ represents the out-of-plane transverse displacement of the tube wall (Fig. 1).

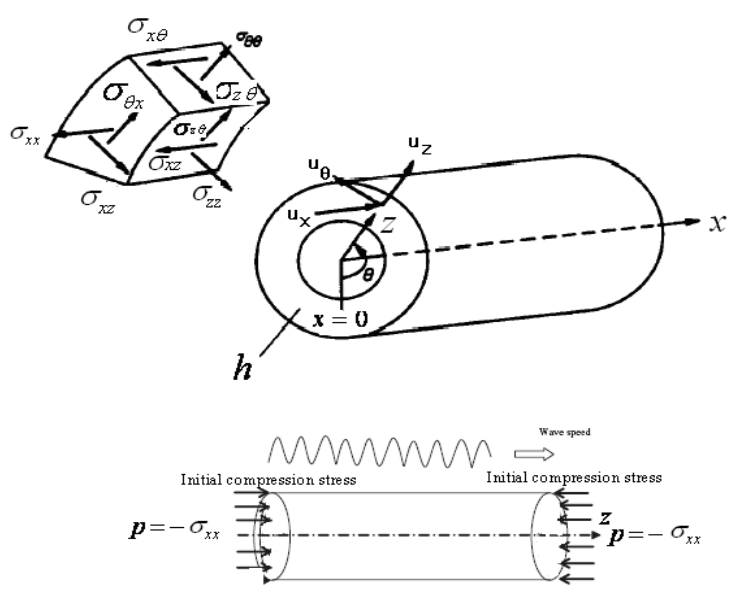

FIG. 1: Definition of geometry of the tube, displacements and stresses.

The relationship of strains and stresses of thin-single tube under initial compression stress can be written as

$$
\left\{\begin{array}{l}
\sigma_{x x} \\
\sigma_{\theta \theta} \\
\sigma_{x \theta}
\end{array}\right\}=\left[\begin{array}{ccc}
c_{11} & c_{12}+P & c_{16} \\
c_{12}+P & c_{22} & c_{26} \\
c_{16} & c_{26} & c_{66}
\end{array}\right]\left(\left\{\begin{array}{c}
s_{x x} \\
s_{\theta \theta} \\
s_{x \theta}
\end{array}\right\}+z\left\{\begin{array}{l}
k_{x x} \\
k_{\theta \theta} \\
k_{x \theta}
\end{array}\right\}\right)
$$

where $P=-\sigma_{z z}$ is the initial stress parameter, $c_{i j}(i, j=$ $1,2,6)$ are the effective elastic constants of single-walled carbon nanotube, $s_{x x}, s_{\theta \theta}, s_{x \theta}$ are the membrane strains of the middle surface, and $k_{x x}, k_{\theta \theta}, k_{x \theta}$ are the curvatures, which are expressed as

$$
\begin{aligned}
& s_{x x}=\frac{\partial u_{x}}{\partial x}, \quad s_{\theta \theta}=\frac{1}{R} \frac{\partial u_{\theta}}{\partial \theta}+\frac{u_{z}}{R}, \quad s_{x \theta}=\frac{1}{R} \frac{\partial u_{x}}{\partial \theta}+\frac{\partial u_{\theta}}{\partial x}, \\
& k_{x x}=-\frac{\partial^{2} u_{z}}{\partial x^{2}}, \quad k_{\theta \theta}=\frac{1}{R^{2}}\left(\frac{\partial u_{\theta}}{\partial \theta}-\frac{\partial^{2} u_{z}}{\partial \theta^{2}}\right), \\
& s_{x \theta}=\frac{1}{R} \frac{\partial u_{\theta}}{\partial x}-\frac{2}{R} \frac{\partial^{2} u_{Z}}{\partial x \partial \theta}
\end{aligned}
$$

where $R$ is the radius of the middle surface of shell. Integrating the stresses across the thickness of the singlewalled carbon nanotube, the membrane forces $N_{x x}, N_{\theta \theta}$ and $N_{x \theta}$ of the middle surface and internal moment $M_{x x}, M_{\theta \theta}$ and $M_{x \theta}$ exerted on the middle surface are expressed as [17]

$$
\left\{N_{x x}, N_{\theta \theta}, N_{x \theta}\right\}=\int_{-h / 2}^{h / 2}\left\{\sigma_{x x}, \sigma_{\theta \theta}, \sigma_{x \theta}\right\} d z,
$$

$$
\left\{M_{x x}, M_{\theta \theta}, M_{x \theta}\right\}=\int_{-h / 2}^{h / 2}\left\{\sigma_{x x}, \sigma_{\theta \theta}, \sigma_{x \theta}\right\} \mathrm{z} d,
$$

where $h$ is the effective thickness of single-walled carbon nanotube. Substitution Eq. (1) into eq. (4), yields

$$
\left\{\begin{array}{l}
N_{x x} \\
N_{\theta \theta} \\
N_{x \theta} \\
M_{x x} \\
M_{\theta \theta} \\
M_{x \theta}
\end{array}\right\}=\left[\begin{array}{llllll}
A_{11} & A_{12} & A_{16} & B_{11} & B_{12} & B_{16} \\
A_{12} & A_{22} & A_{26} & B_{12} & B_{22} & B_{26} \\
A_{16} & A_{26} & A_{66} & B_{16} & B_{26} & B_{66} \\
B_{11} & B_{12} & B_{16} & D_{11} & D_{12} & D_{16} \\
B_{12} & B_{22} & B_{26} & D_{12} & D_{22} & D_{26} \\
B_{16} & B_{26} & B_{66} & D_{16} & D_{26} & D_{66}
\end{array}\right]\left\{\begin{array}{c}
S_{x x} \\
S_{\theta \theta} \\
S_{x \theta} \\
K_{x x} \\
K_{\theta \theta} \\
K_{x \theta}
\end{array}\right\}
$$

where $A_{i j}, D_{i j}$ and $B_{i j}(i, j=1,2,6)$ are the tension stiffness , the bending stiffness and the tension-bending coupled stiffness, which is expressed as [18]

$$
\left\{A_{i j}, B_{i j}, D_{i j}\right\}=\int_{-h / 2}^{h / 2}\left\{1, z, z^{2}\right\} \mathrm{C}_{i j} d z, \quad(i, j=1,2,6) .
$$

The ideal nanotubes structures can be obtained from a rolled graphene sheet with an isotropic material. For isotropic graphene sheet, therefore, the stiffness coefficients in Eq.(4) 
can be simplified and given by

$$
\begin{aligned}
& A_{11}=\left(\frac{\left(1+\zeta_{x}(1-v)\right)}{\left(1-v^{2}\right)}\right) E h, \\
& A_{12}=\left(\frac{v+\zeta_{x}(1-v)}{\left(1-v^{2}\right)}\right) E h, \\
& A_{22}=\frac{E h}{\left(1-v^{2}\right)}, \\
& A_{66}=\frac{E h}{2(1+v)}, A_{16}=A_{26}=0, \\
& D_{11}=\left(\frac{\left(1+\zeta_{x}(1-v)\right)}{\left(1-v^{2}\right)}\right) \frac{E h^{3}}{12}, \\
& D_{12}=\left(\frac{v+\zeta_{x}(1-v)}{\left(1-v^{2}\right)}\right) \frac{E h^{3}}{12},
\end{aligned}
$$

$$
D_{22}=\frac{E h^{3}}{12\left(1-v^{2}\right)}, D_{66}=\frac{E h^{3}}{24(1+v)}, D_{16}=D_{26}=0 .
$$

and the coupled stiffness coefficients $B_{i j}=0$, where $E$ and $v$ are the elastic modulus and is Poisson's ratio of graphene sheet.

Based on Love's first approximation stated above and using the coordinate system defined in Fig. 1, the Flügge type basic equations of motion are proposed as the governing equations of the wave propagation under initial compression stress $\left(\sigma_{x x}=-P\right)$ in CNTs. The equations of motion for the nanotube can be written by [19]

$$
\begin{aligned}
& {\left[A_{11} \frac{\partial^{2}}{\partial x^{2}}+\frac{A_{22}(1-v)}{2 R^{2}} \frac{\partial^{2}}{\partial \theta^{2}}-\rho h \frac{\partial^{2}}{\partial t^{2}}\right] u_{x}+\left[\left(\frac{A_{12}}{R}+\frac{A_{22}(1-v)}{2 R}-\frac{\zeta_{x} E}{2 R(1+v)}\right) \frac{\partial^{2}}{\partial x \partial \theta}\right] u_{\theta}+} \\
& +\left[\left(\frac{A_{12}}{R} \frac{\partial}{\partial x}+\frac{\zeta_{x} E}{2 R^{2}(1+v)} \frac{\partial^{2}}{\partial \theta^{2}}\right)\right] u_{z}=0 \\
& \left(\frac{A_{12}}{R}+\frac{A_{22}(1-v)}{2 R}\right) \frac{\partial^{2}}{\partial x \partial \theta} u_{x}+\left[\left(\frac{A_{22}(1-v)}{2}+\frac{A_{22}(1-v) h^{2}}{24 R^{2}}-\frac{\zeta_{x} E}{2(1+v)}\right) \frac{\partial^{2}}{\partial x^{2}}+\left(\frac{A_{22}}{R^{2}}+\frac{h^{2} A_{22}}{12 R^{4}}\right) \frac{\partial^{2}}{\partial \theta^{2}}-\rho h \frac{\partial^{2}}{\partial t^{2}}\right] u_{\theta} \\
& +\left[\left(\frac{A_{22}}{R^{2}} \frac{\partial}{\partial \theta}-\frac{h^{2} A_{22}}{12 R^{4}} \frac{\partial^{3}}{\partial \theta^{3}}+\frac{\zeta_{x} E}{2 R(1+v)} \frac{\partial^{2}}{\partial x \partial \theta}-\left(\frac{h^{2} A_{22}}{6 R^{2}}+\frac{h^{2} A_{12}}{12 R^{2}}\right) \frac{\partial^{3}}{\partial x^{2} \partial \theta}\right)\right] u_{z}=0, \\
& {\left[-\frac{A_{12}}{R^{2}} \frac{\partial}{\partial x}\right] u_{x}+\left[\left(\frac{h^{2} A_{12}}{12 R^{2}}+\frac{A_{22}(1-v) h^{2}}{12 R^{2}}\right) \frac{\partial^{3}}{\partial x^{2} \partial \theta}+\left(\frac{h^{2} A_{22}}{12 R^{4}}\right) \frac{\partial^{3}}{\partial \theta^{3}}-\left(\frac{A_{22}}{R^{2}}\right) \frac{\partial}{\partial \theta}\right] u_{\theta}} \\
& \quad-\left[\frac{h^{2} A_{11}}{12} \frac{\partial^{4}}{\partial x^{4}}+\left(\frac{h^{2} A_{12}}{6 R^{2}}+\frac{A_{22}(1-v) h^{2}}{6 R^{2}}\right) \frac{\partial^{4}}{\partial x^{2} \partial \theta^{2}}+\frac{h^{2} A_{22}}{12 R^{4}} \frac{\partial^{4}}{\partial \theta^{4}}+\frac{\zeta_{x} E}{2(1+v)} \frac{\partial^{2}}{\partial x^{2}}+\frac{A_{22}}{R^{2}}+\rho h \frac{\partial^{2}}{\partial t^{2}}\right] u_{z}+\hat{P}=0
\end{aligned}
$$

where $\zeta_{x}=\frac{P(1+v)}{E}, h$ is the thickness of the CNT, pis the mass density, $P$ is the initial compression stresses, and $\hat{P}$ is the pressure between two adjacent nanotubes ( mainly due to the van der Waals interaction).

In matrix form, equations (7) may be expressed as:

$$
\left[\begin{array}{ccc}
L_{11}+\alpha_{11} & L_{12}+\alpha_{12} & L_{13}+\alpha_{13} \\
L_{12}+\alpha_{21} & L_{22}+\alpha_{22} & L_{23}+\alpha_{23} \\
-L_{13}+\alpha_{31} & -L_{23}+\alpha_{32} & L_{33}+\alpha_{33}
\end{array}\right]\left[\begin{array}{c}
u_{x} \\
u_{\theta} \\
u_{z}
\end{array}\right]=\left[\begin{array}{c}
0 \\
0 \\
-\beta \hat{P}
\end{array}\right],
$$

where $L_{i j}(i, j=1,2,3)$ are the differential operators with respect to $x$ and $\theta$, given by

$$
\begin{aligned}
L_{11} & =\frac{\partial^{2}}{\partial x^{2}}+\frac{1-v}{2 R^{2}} \frac{\partial^{2}}{\partial \theta^{2}}-\beta \rho h \frac{\partial^{2}}{\partial t^{2}}, \\
L_{12} & =\frac{1+v}{2 R} \frac{\partial^{2}}{\partial x \partial \theta}, L_{13}=\frac{v}{R} \frac{\partial}{\partial x} \\
L_{22} & =\frac{1-v}{2} \frac{\partial^{2}}{\partial x^{2}}+\frac{1}{R^{2}} \frac{\partial^{2}}{\partial \theta^{2}}+\sigma \\
& \times\left((1-v) \frac{\partial^{2}}{\partial x^{2}}+\frac{1}{R^{2}} \frac{\partial^{2}}{\partial \theta^{2}}\right)-\beta \rho h \frac{\partial^{2}}{\partial t^{2}},
\end{aligned}
$$

$$
\begin{gathered}
\mathrm{L}_{23}=\frac{1}{R^{2}} \frac{\partial}{\partial \theta}-\sigma\left((2-v) \frac{\partial^{3}}{\partial x^{2} \partial \theta}+\frac{1}{R^{2}} \frac{\partial^{3}}{\partial \theta^{3}}\right), \\
L_{33}=-\frac{1}{R^{2}}-\sigma\left(R^{2} \frac{\partial^{4}}{\partial x^{4}}+2 \frac{\partial^{4}}{\partial x^{2} \partial \theta^{2}}+\frac{1}{R^{2}} \frac{\partial^{4}}{\partial \theta^{4}}\right)-\beta \rho h \frac{\partial^{2}}{\partial t^{2}},
\end{gathered}
$$

and

$$
\begin{aligned}
\alpha_{11} & =\zeta_{x}(1-v) \frac{\partial^{2}}{\partial x^{2}}, \alpha_{12}=\frac{\zeta_{x}(2 h-1)(1-v)}{2 R} \frac{\partial^{2}}{\partial x \partial \theta} \\
\alpha_{13} & =\frac{\zeta_{x}(1-v)}{R}\left(\frac{\partial}{\partial x}+\frac{1}{2 R h} \frac{\partial^{2}}{\partial \theta^{2}}\right)
\end{aligned}
$$

$$
\begin{aligned}
\alpha_{21} & =\zeta_{x}(1-v) \frac{\partial^{2}}{\partial x \partial \theta}, \alpha_{22}=\frac{\zeta_{x}(1-v)}{2 h} \frac{\partial^{2}}{\partial x^{2}}, \\
\alpha_{23} & =\frac{\zeta_{x}(1-v)}{2 h R}\left(\frac{\partial^{2}}{\partial x \partial \theta}-\frac{h^{3}}{6 R} \frac{\partial^{3}}{\partial x^{2} \partial \theta}\right) \\
\alpha_{31} & =\frac{\zeta_{x}(1-v)}{R} \frac{\partial}{\partial x}, \alpha_{32}=\frac{\zeta_{x} h^{2}(1-v)}{12 R} \frac{\partial^{3}}{\partial x^{2} \partial \theta}, \\
\alpha_{33} & =\frac{\zeta_{x}(1-v)}{2 h}\left(\frac{\partial^{2}}{\partial x^{2}}+\frac{h^{3}}{6} \frac{\partial^{4}}{\partial x^{4}}+\frac{h^{3}}{3 R^{2}} \frac{\partial^{3}}{\partial x^{2} \partial \theta}\right),
\end{aligned}
$$


$\sigma=\frac{h^{2}}{12 R^{2}} \quad$ is the nondimensional thickness parameter, and

$\beta=\frac{\left(1-v^{2}\right)}{E h}$.

Equation (8) represents a set of three expressions as:

$$
\begin{aligned}
& D_{11} u_{1}+D_{12} u_{2}+D_{13} u_{3}=0, \\
& D_{21} u_{1}+D_{22} u_{2}+D_{23} u_{3}=0, \\
& D_{31} u_{1}+D_{32} u_{2}+D_{33} u_{3}+\beta \hat{P}=0,
\end{aligned}
$$

where

$$
\begin{aligned}
& D_{11}=L_{11}+\alpha_{11}, D_{12}=L_{12}+\alpha_{12}, D_{13}=L_{13}+\alpha_{13}, \\
& D_{21}=L_{12}+\alpha_{21}, D_{22}=L_{22}+\alpha_{22}, D_{23}=L_{23}+\alpha_{23}, \\
& D_{31}=-L_{13}+\alpha_{31}, D_{32}=-L_{23}+\alpha_{32}, D_{33}=L_{33}+\alpha_{33} .
\end{aligned}
$$

\section{TORSIONAL VIBRATION OF CARBON NANOTUBE}

For the present problem we confine ourselves to study a torsional vibration of carbon nanotubes. Assuming that a nanotube vibrates in torsion only, such that the cross-section of the tube is not elastically deformed, we can set

$$
u_{x}=u_{z}=0 \text { and } \frac{\partial(.)}{\partial \theta}=0
$$

Following the usual procedure for the problems having $\theta$ symmetry and using (13) it can easily be seen that the remaining part of equation of motion (7) becomes

$$
\begin{aligned}
& \left(\frac{A_{22}(1-v)}{2}+\frac{A_{22}(1-v) h^{2}}{24 R^{2}}-\frac{\zeta_{x} E}{2(1+v)}\right) \\
& \frac{\partial^{2} u_{\theta}}{\partial x^{2}}-\rho h \frac{\partial^{2} u_{\theta}}{\partial t^{2}}=0
\end{aligned}
$$

Using Eq.(6) into Eq.(14) one get

$$
\begin{aligned}
& \left(\frac{E h}{2(1-v)}+\frac{E h}{2(1-v)}\left(\frac{h^{2}}{12 R^{2}}\right)-\frac{\zeta_{x} E}{2(1+v)}\right) \\
& \frac{\partial^{2} u_{\theta}}{\partial x^{2}}-\rho h \frac{\partial^{2} u_{\theta}}{\partial t^{2}}=0
\end{aligned}
$$

Since the shear modulus $G=\frac{E}{2(1+v)}$, thus the equation of motion(15) becomes

$$
\left(G\left(1+\frac{h^{2}}{12 R^{2}}\right)-\frac{\zeta_{x} E}{2 h(1+v)}\right) \frac{\partial^{2} u_{\theta}}{\partial x^{2}}-\rho \frac{\partial^{2} u_{\theta}}{\partial t^{2}}=0
$$

Since we may assume that $h^{2} / 12<<1$, and introducing the velocity of shear sound wave at the initial stress-free $\left(\zeta_{x}=0\right)$

$$
\alpha=\sqrt{\frac{G}{\rho}}
$$

We obtain

$$
\frac{\partial^{2} u_{\theta}}{\partial t^{2}}-\left(\alpha^{2}-\frac{\zeta_{x} E}{2 h \rho(1+v)}\right) \frac{\partial^{2} u_{\theta}}{\partial x^{2}}=0
$$

\section{PARTICULAR CASE}

The presence of $\zeta_{x}$ in relation (18) shows that the effect of initial stress on the equation of motion of nanotube in torsion. If the nanotube is free from initial stresses, $\left(\zeta_{x}=0\right)$, the equation of motion (18) becomes

$$
\frac{\partial^{2} u_{\theta}}{\partial t^{2}}-\alpha^{2} \frac{\partial^{2} u_{\theta}}{\partial x^{2}}=0
$$

which coincide with the result given by Werner [20] at the absent of external forces.

\section{PHASE VELOCITY OF TORSIONAL WAVES}

Assuming a harmonic wave solution, namely

$$
u_{\theta}(x, z, t)=A(z) \exp [i(k x-\omega t)],
$$

where $A(z)$ is the amplitude of the wave propagation, and $\omega$ is the circular frequency of the wave. Substitution of (20) into (18) leads to:

$$
k^{2}\left(\beta^{2}-\frac{\zeta_{x} E}{2 h \rho(1+v)}\right)-\omega^{2}=0
$$

where $k \mathrm{i} \mathrm{s}$ the wave number which is related to the angular frequency by

$$
k=\omega / c .
$$

and $\rfloor$ is the phase velocity of torsional wave. Inserting Eqs.(17) and (22) into Eq.(21) the phase velocity (c) of torsional wave may be obtained as

$$
\frac{C}{\beta}=\sqrt{1-\frac{\zeta_{x} E}{2 h G(1+v)}}
$$

A negative sign before the term $\frac{\zeta_{x} E}{2 h G(1+v)}$ of relation (23) indicates that the presence of initial compression stresses $\left(\zeta_{x}\right)$ reduce the phase velocity $c$, which is the same as obtained by Narian [21]

\section{NUMERICAL RESULTS AND DISCUSSION}

The purpose of numerical computation is to examine the effect of compression initial stresses on phase velocity of torsional waves propagating in SWCNTs. In order to perform numerical calculation from equation (23) the CNTs to be considered has the elastic modulus of graphene sheets with $E=1.095 T P a$, Poisson's ratio of $v=0.19$ and shear modulus of $G=0.4601 \mathrm{TPa}$. The mass density of CNTs of $\rho=1.3 \mathrm{~g} / \mathrm{cm}^{3}$. The SWCNTs are $(40,0)$ zigzag tubes and has a diameter of $R=2.0 \mathrm{~nm}$.

The dispersion curves of dimensionless phase velocity of torsional waves with different values of thicknesses(nanometer) of SWCNTs under initial compression stresses are presented in figure (2). The results show that ashincreases the phase velocity of torsional wave increase. 


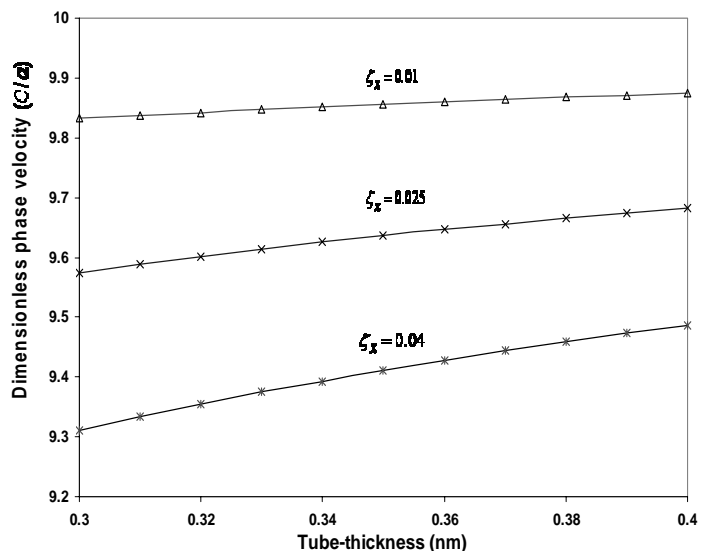

FIG. 2: Variation of dimensionless phase velocity $(C / \alpha)$ with tube-thickness $(h)$ for different values of initial stress parameter $\zeta_{x}=0.01,0.025,0.04$.

In order to examine the influence of the initial compression stresses on the torsional wave propagation of SWCNTs, the results under initial compression stress $\zeta_{x}=0.01$, $\zeta_{x}=0.025$ and $\zeta_{x}=0.04$ are plotted in figure (2). Comparisons show that the change tendency of the wave speeds of torsional waves in single-walled CNTs with initial compres- sion stresses $\zeta_{x}=0.025$ is similar to those in at $\zeta_{x}=0.01$, and the change is clearly observed in the case of $\zeta_{x}=0.04$. The curves reveal clearly that the presence of initial compression stresses affects the propagation of torsional wave in sense that every small change in the value of initial stress parameter $\zeta_{x}$ produces a substantial change in the phase velocity.

\section{CONCLUSIONS}

It is concluded that the presence of initial compression stresses has a notable effect on the propagation of torsional waves in single-walled carbon nanotubes. The study also, shows that the presence of compressive initial stresses decreases the velocity of torsional waves propagating in singlewalled carbon nanotubes. The investigation presented may be helpful in the application of CNTs, such as high - frequency oscillators and mechanical sensors.

\section{Acknowledgment}

The author wish to acknowledge support provided by the King Saud University and the Aflaj community college. Also, the author are thankful to Dr. Rashed Al-Rushood (dean of the Aflaj community college) for his supporting and encouragement during this work.
[1] S. Iijima, Helical microtubules of graphitic carbon, Nature 354(1991) pp. 56-58.

[2] D. Qian, J.G. Wagner, W.K. Liu, M.F. Yu and R.S. Ruoff, Mechanics of carbon nanotubes, Appl Mech Rev 55 (2002) (6), pp. 495-533.

[3] P. Antonio, M. P. David , C. B. Mary, Mechanics of deformation of single- and multi-wall carbon nanotubes, Journal of the Mechanics and Physics of Solids 52 (2004) $789-821$.

[4] T. Natsuki , M. Endo and H. Tsuda 2006 J. Appl. Phys. 99 034311.

[5] W. Flügge Statik und Dynamik der Schalen. Berlin: Springer(1934).

[6] M. M. Selim, Vibrational analysis of carbon nanotubes under initial compression stresses, NANO Conference 2009, April 5-7, 2009, King Saud University, KSA.

[7] Y. Zhang, G. Liu and X. Han, X 2005 Phys. Lett. A 340 258266.

[8] H. Zhang and X. Wang, Effects of initial stress on transverse wave propagation in carbon nanotubes based on Timoshenko laminated beam models, Nanotechnoology 17(2006), pp.4553.

[9] X. Wang and H. Cai , Effects of initial stress on non-coaxial resonance of multi-wall carbon nanotubes, Acta Mater. 54 (2006), pp.2067-2074.

[10] K. Liu and C. Sun, Vibration of multi-walled carbon nanotubes with initial axial loading, Solid State Communications 143 (2007), pp. 202-207.

[11] X. Chen and X. Wang, Effects of initial stress on wave propagation in multi-walled carbon nanotubes Phys. Scr. 78 (2008)
015601 (9pp).

[12] Rayleigh, The theory of sound, Dover, New York (1945).

[13] R. C. Bhattacharya, On the torsional wave propagation in a two-layered circular cylinder with imperfect bonding. Proc. Indian natn. Sci. Acad., 41, A, No. 6 (1975) PP. 613-619.

[14] H. S. Paul and K.V. Sarma, Torsional wave propagation in a finite piezoelectric cylindrical shell. Proc. Indian natn. Sci. Acad., 43, A, No.2(1977)pp. 169-181.

[15] S. Dey and A. Dutta, Torsional wave propagation in An initially stressed cylinder. Proc. Indian natn. Sci. Acad., 58, A, No. 5(1992) pp.425429.

[16] M. M. Selim, Torsional waves propagation in an initially stressed dissipative Cylinder. Applied Mathematical Sciences ,Vol. 1,no.29(2007) pp. 1419-1427.

[17] A. W. Leissa , Vibration of shells (Scientific and Technical Information Office, National Aeronautics and Space Administration, Washington (1973).

[18] K. Dong, B. Y. Liu and X. Wang, Wave propagation in fluidfilled multi-walled carbon nanotubes embedded in elastic matrix. Computational Material Science 42 (2008) pp. 139-148.

[19] M. M. Selim , S. Abi and K. Harigaya , Effects of initial compression stress on wave propagation in carbon nanotubes, Eur. Phys. J. B 69, 523-528 (2009).

[20] W. Soedel, Vibration of shells and plates, Marcel Dekker, Inc. Third Edition, New York, (2004) pp. 74

[21] S. Narian , Magnetoelastic torsional vibration of nonhomogenous aeolotropic cylindrical shell of viscoelastic solids, Def. Sci. J, Vol. 54 ,No.4 (2004) pp. 443-454. 